\title{
The grand challenges in marine pollution research
}

\author{
Hans U. Dahms* \\ Department of Biomedical Science and Environmental Biology, Kaohsiung Medical University, Kaohsiung, Taiwan \\ *Correspondence: hansd@kmu.edu.tw; marc436@yahoo.com
}

Edited and reviewed by:

Sami Souissi, University of Lille 1, France

Kyung-Hoon Shin, Hanyang University, South Korea

Keywords: oceanic pollution, stressor, integration, multidisciplinary, systems approach, novelty, innovation

\section{INTRODUCTION}

Given the importance of oceans to humankind and the increasing pressure they are under, it is timely to identify and prioritize oceanic health issues that are covered in their ill-defined state by "marine pollution" (MP). MP increasingly leads to disturbances of the oceanic environment and its biota and adversely affects environmental and human health. Pollutants may have various biological impacts such as death, metabolic malfunction, genetic and phenological damage. If such impacts are sublethal, they will lead to fitness changes. Depleted numbers of sensitive species are causing a decrease in biodiversity, and may cause ecosystem function changes by habitat and food chain alterations and those of productivity patterns.

Major challenges in MP studies are conceptional as well as operational. Conceptionally, pollutants are very much understood as chemicals only. From a largely chemical perspective pollution studies need to open up to any stressor that affects organisms in their respective environment. Stress to organisms in the marine environment can be caused by physical (e.g., electromagnetic radiation, electricity, drag etc.) chemical (e.g., organic or inorganic), physico-chemical $(\mathrm{pH})$ or biological factors (biotoxins, competition, predation, parasitism). MP is also very much perceived as man-made although there exists natural pollution since ever-if natural is understood as stressors of organisms that are not anthropogenic (e.g., input of freshwater, sediments and their contaminants, volcanic activity outside and inside the oceans). Natural pollution happened even before humans contributed to MP so much more in recent centuries.
Humans intensified natural MP and certainly created novel stressors through technological innovations. These got magnified as a new quality in the antropocene by man-made changes to soil, atmosphere and waters. The hydrosphere includes the oceans which cover about $70 \%$ of the earth's surface and are providing more than $99 \%$ of the earth's water resources. Man-made effects include industrial (e.g., noise, radiation, heavy metals, nanoparticles), agricultural (e.g., pesticides, antibiotics, fertilizers), and urban pollutants (e.g., organic matter, pharmaceuticals, $\mathrm{CO}_{2}$ ) which reach the oceans via various pathways, from the atmosphere, aquatic drainages and rivers, from coastal groundwater, and through organisms getting dispersed between these realms (Sweet, 2013). Everything that humans are doing will have consequences. Human activities are never environmentally neutral.

As in all sciences it will be important to make temporal and spatial distinctions in MP studies. Spatially-the oceans are not separated from other realms, such as land and freshwater systems and the atmosphere. Multiple interfaces facilitate the fluxes of energy and matter that also allow the influx of stressors. Within the oceans there are interactions between sea bottom and water column, and water column and atmosphere (or seasonal and multiyear sea-ice and atmosphere during winter and in polar seas). Distribution patterns of stressors may exhibit substantial horizontal and vertical patchiness. Several characteristics of the sea surface can remotely be monitored meanwhile by Geographic Information Systems (GIS) approaches. Temporally_-stressors may act at a gradient of very different time scales: from geological times shaping the adaptation and evolution of organisms (Dahms and Lee,
2010), to minutes and seconds demarcating behavior (Michalec et al., 2013), and even to parts of a second where chemical reactions typically take place (Dahms et al., 2014).

There is a distinction between field and laboratory approaches. Field-oriented approaches are acting at the natural in situ platform where stressors originate or might get transformed, disposed and remobilized. Field approaches are often seen inferior to laboratory in vitro approaches. The latter are expected to provide a better experimental and analytical resolution. It is a major challenge and strongly pleaded here to integrate both approaches in order to obtain a more realistic understanding about the mechanism of action in the natural world which we are ultimately concerned with. Besides taken field samples from natural or experimental sites to the laboratory for further study there is the possibility of micro- to mesocosm studies which provide a gradient from strictly controlled experiments to increasingly complex interaction of various variables that are characteristic for the real world. The main challenge here will be to study the interactions at interphases multidisciplinary and integrate the results in a systems approach (Dahms and Lee, 2010).

In the following I will demarcate challenges which are provided by some nonexhaustive examples of novel stressors in oceanic pollution studies followed by challenges to novel approaches to various aspects of MP. At the same time there are challenges for innovative approaches to analyze sources, mechanisms of actions, and effects of stressors. These will provide new options for remediation, education, management, and innovative policies for the health management of the oceans. 


\section{CHALLENGES FROM NOVEL STRESSORS \\ CLIMATE CHANGES}

Climate changes are impacting global water resources, and increasing the need for a deeper understanding of the interaction between climate and natural resources. Given the global climate challenge this will affect non-chemical stressors (habitat loss, invasive species), and will affect chemical toxicity alike (climate-induced toxicity susceptibility, toxicant-induced climate susceptibility). Observational palaeolimnological techniques such as the use of microfossils (forams, diatoms, pollen etc.) are well established and can significantly contribute to the understanding of climatic variability and the impacts that change in climate have on marine ecosystems. These can utilize a combination of proxies including a range of biological fossils and metagenomic, and geochemical chronological techniques to investigate long-term climate changes in the marine realm (Hembrow et al., 2014). There is a great concern for both MP and Global Climate Change, but the interaction between both forcing factors on marine ecosystems is not deeply studied. Climate Change may increase the vulnerability (or stress level) of several marine organisms/ecosystems that makes MP also a serious issue in the Global Change discussion (Lelieveld et al., 2001).

\section{MICROPLASTICS}

Marine debris is a growing global problem posing a threat to a variety of marine organisms through toxic action of nanoparticles and the ingestion of particles and entanglement. Plastics are the most common type of marine debris, constituting between 60 and $80 \%$ of all marine debris and over $90 \%$ of all floating particles. Particularly microplastics are of concern because they can be ingested by a variety of marine organisms, and possibly can also be transferred within food webs. The potential toxicity of microplastics is basically due to the additives and monomers they include. Microplastics can effectively absorb hydrophobic contaminants from the water due to their relatively large area to volume ratio. Experiments were carried out with different Baltic Sea zooplankton taxa to scan their potential to ingest plastics. These demonstrated the ingestion of microspheres in all the taxa studied, from lower (mesozooplankton) to higher trophic level (macrozooplankton) (Setälä et al., 2014).

\section{PHARMACEUTICALS}

Among the emerging contaminants, are pharmaceuticals one of the most relevant groups of substances in aquatic ecosystems due to universal use, their chemicophysical properties and unknown mode of action in aquatic organisms at low concentrations. After administration many drugs and their transformation products are retained only to some extent in wastewater treatment plants. They then enter the aquatic environment in considerable high amounts. The annual drug consumption in treating human and animal diseases, also in livestock and aquaculture, was estimated to be thousands of tons worldwide (WHO, 2012). This was leading to high concentrations in surface water also of developed countries. Chronic and subtle effects can be expected when aquatic organisms are exposed to persistent and accumulative compounds for longterm. Aspects of bioconcentration, bioaccumulation and potential biomagnification in aquatic ecosystems are unknown not only for most pharmaceuticals but other compounds as well. More comprehensive assessments for the evaluation of environmental and human health risks and analytical methods are required to detect the bioaccumulation of pharmaceuticals (see Zenker et al., 2014).

\section{NOISE}

The World Health Organization has recently acknowledged that contrary to the trend for other environmental stressors, noise exposure is increasing worldwide. Since the establishment of the European Noise Directive in 2002, there has been a significant improvement in the awareness among the general public and policymakers about the relationship between human exposure to environmental noise and related public health concerns (Murphy and King, 2014). As a result, the importance of environmental noise pollution in shaping urban, environmental and public health policies is increasing internationally. Health issues associated with noise pollution are now fairly well-established and extensively documented. In fact, recent research suggests that chronic exposure to environmental noise can lead to a permanent disruption in sleep. This may hold not only for humans since noise disturbs other marine organisms as well with effects on several levels of integration.

\section{COMBINED EFFECTS, BIOACCUMULATION, AND BIOMAGNIFICATION}

Bioavailable stressors in combination may have other than their added single effects in MP studies. The interaction of multiple elements in a system may produce an effect different from or greater than the sum of their individual effects, i.e., synergy. Pollutants also commonly bioaccumulate in individuals and their organs differentially and may get biomagnified through trophic cascades in food webs. Here top predators should particularly be taken into account, but also data concerning the stressor distribution in the whole aquatic environment (water, sediment, biota) should be measured or collected for an effect evaluation of bioaccumulation and biomagnification. When tissue residues are analyzed there should be a focus on the toxicodynamics (action and potency) of the toxicants as well as on toxicokinetic variations (temporal aspects of accumulation, biotransformation, and internal distribution) (Zenker et al., 2014).

\section{INNOVATIVE APPROACHES IN MP-SCIENCE, TECHNOLOGY, POLICIES}

There are new approaches needed in order to face new challenges in monitoring the presence and effects of stressors and in the analytical study of mechanisms-of-action. Some novel approaches are readily available that have to be taken to practice and evaluated in their efficiency. Here, some challenging technologies are briefly summarized as examples.

\section{INNOVATIVE CHALLENGES IN THE FIELD}

GIS have gained popularity in recent years because they provide spatial data management and access through the web. Ocean surveillance has traditionally been accomplished by aircraft and coastguard forces, whose work, unfortunately, is limited by the monitoring costs of large areas. In this respect, spacecrafts provide a better solution. Today we can observe 
ocean pollution in the shape of massive plastic stains or oil carpets resulting from grounded tankers or the harvest of crude oil. Regarding the type of sensors used in ocean monitoring, microwaves are preferred to optical sensors, as they can provide data under all weather and brightness conditions. These type of sensors are called Synthetic Aperture Radars (SAR), which capture the scatter of microwaves on a determined surface and are optimal for capturing the roughness of the sea surface, regardless of weather and light conditions. Fustes et al. (2014) provide an exemplary tool that offers an integrated framework for the detection and localization of marine spills using remote sensing, GIS, and cloud computing. The authors present advanced segmentation algorithms are presented in order to isolate dark areas in SAR images, including fuzzy clustering and wavelets.

To assess environmental hazards, such as of persistent organic pollutants (POPs) and EDCs to marine organisms, it is important to examine the occurrence, fate, and distribution of contaminants in both sediment and water column from physical samples (Lee et al., 2013). Instrumental analysis, however, is usually time consuming and expensive due to the exhaustive clean-up required to remove all the interferences and the use of sophisticated and expensive instruments. Due to the complex nature of environmental mixtures, it is further difficult to predict potential effects of environmental contaminants based on instrumental analysis alone. Such shortcomings led to the development of new and alternative methods which apply biological techniques to determine these compounds. As an example for such innovations provides the sediment quality triad approach an effect-based approach that combines measures of various chemicals, potential toxicities, and benthic community structures (Khim and Hong, 2014).

\section{OMICs-APPROACHES}

OMICs studies refer to modern biological approaches ending in -omics, such as genomics, proteomics or metabolomics. The related suffix -ome addresses the objects of study, such as the genome, proteome or metabolome, respectively. OMICs studies aim at the collective characterization and quantification of pools of biological molecules and a bioinformatic evaluation of those that translate into the structure, function, and dynamics of organisms. In MP studies toxicogenomics pursue how toxicants interact with genetic material (Dahms and Lee, 2010). Stressors can cause genotoxic effects which alter genetic material which in turn generates irreversible damage or mutations, with consequences at all downstream integration levels of organisms. Massive DNA damage can promote death or sterility in individuals, whereas other sublethal gene mutations may change the germ line and this way affect the gene pool of a population. If sublethal this can lead to microevolutionary processes. Evolutionary adaptations will select pollution-tolerant individuals that then change the genetic diversity of populations (Piña and Barata, 2014).

Formerly, large-scale proteomics was only possible for model organisms whose genomes were sequenced. The use of nextgeneration sequencers is now changing this scenario. Proteogenomics meanwhile allows the use of experimental data to refine genome annotations. Combining genomic and proteomic data is becoming routine in many research projects. Genome drafts can be retrieved for any organism using next-generation sequencers at reasonable cost. The use of RNA-seq to establish nucleotide sequences that are directly translated into protein sequences appears to be a promising challenge (Armengaud et al., 2014).

\section{MODELING APPROACHES}

Marine system models can provide useful predictions. Experts generally agree that ecological models only provide predictions of real system functioning when there are strong physical (as opposed to chemical or ecological) drivers. Recent developments in modeling include changes in technology, changes in the modeling community and changes in the context in which modeling is conducted. According to Robson (in press) do current trends increase the data assimilation, operationalization, the integration of models, and the development of improved tools for skill assessment. The author claims that a merge of mechanistic and stochastic modeling through approaches like Bayesian Hierarchical Modeling and Bayesian Melding or surrogate modeling are understood as key emerging areas.

\section{POLICIES}

MP creates an economic problem since it reduces the value of the natural capital provided by oceanic resources. At present the financial system tends to look only toward immediate profit, discounting ecosystem services and medium and long term advantages of environmental protection vs. treatment of damages that have occurred. It may be challenging to reevaluate the practice of protection with respect to the precautionary principle (Wiegleb et al., 2013). There is a policy problem in the sense that we need to allocate economic resources for the transition and to consider also the social transformations that it will cause. We need to build good models that can suggest us where to go and what measures to take. Such diagnosis should enable identification of appropriate tools, information products, and relationships that can facilitate our goals (see Spruijt et al., 2014).

\section{ROLE OF SCIENTISTS, EDUCATORS, PRACTITIONERS}

What other challenges are there for science in the transition to a modern approach of oceanic health studies? Traditionally, scientists have been required to study and develop new and improved technologies in MP studies: better monitoring approaches, better remediation, better management of MP that provide more efficient ways to use investments of man-power, materials and know-how. These are valid approaches, but more may be needed for a transition in MP. We need to develop and evaluate new technologies in the monitoring, mechanistic studies, and the remediation of the oceans in terms of efficiency, their own environmental impact, and their impact on other economic activities. From there, we need to develop strategies to optimize technological benefits and minimize their unintended negative effects-such as those of treatments of polluted areas or organisms. Scientists have the background and responsibility to understand MP as members of a connected global society. With this understanding a further responsibility as citizen scientists is to 
engage others in deliberative discussions on scientific effords and outcomes, to take actions ourselves in order to mitigate human-caused MP.

Sustainable development activities may comprise of complex sets of ecological, social, economic, and ecological factors. Cross-scale knowledge and applications are increasingly valuable today in achieving successful interdisciplinary action. This also holds for research collaborations among universities and other stakeholders in order to understand and manage the transformative changes in the context of MP. Three aspects may be useful for putting this into an educational practice: (1) making analytical approaches and integrative tools more useful for students, university educators, researchers and academic-practitioners, to incorporate them into interdisciplinary curricula, teaching, research and practice; (2) offering such tools to facilitate integrative action research and collaborative partnerships among educators, researchers, and other academic practitioners; (3) demonstrating how a holistic synthesis can enrich a systems approach to problems such as MP.

For an integrative, multidisciplinary systems approach to MP there are substantial applications for academia, society and industry: e.g., fundamental research in various fields, environmental management (pollution, toxicology, conservation); public health (groundand recreational waters, environmental hygiene, epidemiology); industrial ecology (natural conservation \& restoration, antifouling, aquaculture, food, effect and side-effect screening of pharmaceuticals, cosmetics, and any other industrial products that need to proof safety to human and environmental health). If we can respond to such scientific and technological challenges and transfer scientific and technological knowledge into innovative policies at the national and international level, we can eventually restore a cleaner ocean with its infinite resources and a more equitable and sustainable future for all.

\section{CONCLUSIONS}

Monitoring and assessments of the marine environmental health status should become integral components of adaptive management programs that are aimed to monitor and remediate MP and the damage it causes to the oceanic environment. Such effords taken separately may not be sufficient for detecting unwanted changes of integrative ecosystem health in a complex marine environment. Complexity is here provided by spatio-temporal gradients, such as geographic, latitudinal, depth, as well as seasonal shifts. In addition, organisms show commonly variable reactions at various levels of integration (e.g., at the level of genome and proteome, physiology, cell, tissue and organ, individual, population, and community). Biota are also characterized by variability in their taxonomic and ontogenetic sensitivity, and different reaction norms of sex. The tendency of most toxicants for differential individual bioaccumulation and biomagnification within food webs further complicates the situation. To date, only a few attempts have been made to challenge an integrative approach using, physical and chemical habitat assessments, biological monitoring, and physiological, biochemical, and genotoxicological parameters to assess the environmental health status of a contaminated aquatic ecosystem. In order to integrate abiotic and biotic endpoints, different approaches should be pursued in a systems-oriented way: physical, chemical, biological; laboratory vs. field; realms (freshwater, brackish, marine-bottom, water column, interfaces); organisms (producer, consumer, decomposer); biological integration levels (ecological, behavioral, chemical, and subcellular). This holds for observational monitoring as well as for experimental approaches at all integrations levels-from molecules to ecosystems. Descriptions on disposal patterns of the past and the present need to be considered as well as explanations of the effects and mechanisms of action of natural and man-made pollutants generally. The interactions of stressors in the marine realm at interfaces with atmosphere, land and freshwaters are of particular multidisciplinary interest. Challenges are provided at most levels of MP: pollution monitoring, treatment, and management, economicial, social, and policy aspects in the protection of the marine environment at national and international levels.

\section{ACKNOWLEDGMENT}

This work was supported by a collaboration grant of NRF (2012R1A2A2A 02012617).

\section{REFERENCES}

Armengaud, J., Trapp, J., Pible, O., Geffard, O., Chaumot, A., and Hartmann, E. M. (2014). Nonmodel organisms, a species endangered by proteogenomics. J. Proteomics. doi: 10.1016/j.jprot. 2014.01.007. [Epub ahead of print].

Dahms, H.-U., and Lee, J.-S. (2010). Molecular effects and responses to UV radiation in aquatic ectotherms. Aquatic Toxicology 97, 3-14. doi: 10.1016/j.aquatox.2009.12.002

Dahms, H.-U., Tseng, L.-C., Shim, D. M.-C., and Hwang, J.-S. (2014). Hydrothermal vent effluents affect life stages of the copepod Tisbe sp. J. Mar. Sci. Technol. 22, 82-88. doi: 10.6119/JMST-013-0312-2

Fustes, D., Cantorna, D., Dafonte, C., Arcay, B., Iglesias, A., and Manteiga, M. (2014). A cloudintegrated web platform for marine monitoring using GIS and remote sensing. Application to oil spill detection through SAR images. Future Generation Comput. Syst. 34, 155-160. doi: 10.1016/j.future.2013.09.020

Hembrow, S. S., Taffs, K. H., Atahanb, P., Parr, J., Zawadzki, A., and Heijnis, H. (2014). Diatom community response to climate variability over the past 37.000 years in the sub-tropics of the Southern Hemisphere. Sci. Total Environ. 468-469, 774-784. doi: 10.1016/j.scitotenv. 2013.09.003

Khim, J. S., and Hong, S. (2014) Assessment of trace pollutants in Korean coastal sediments using the triad approach: a review. Sci. Total Environ. 470-471, 1450-1462. doi: 10.1016/j.scitotenv.2013.07.052

Lee, D.-H., Kim, J.-H., Bahk, J.-J., Cho, H.-Y., Hyun, J.-H., and Shin, K.-H. (2013). Geochemical signature related to lipid biomarkers of ANMEs in gas hydrate-bearing sediments in the Ulleung Basin, East Sea (Korea). Mar. Petroleum Geol. 47, 125-135. doi: 10.1016/j.marpetgeo.2013.06.003

Lelieveld, J., and Crutzen, P. J., Ramanathan, V. et al. (2001). The Indian ocean experiment: widespread air pollution from South and Southeast Asia. Science 291, 1031-1036. doi: 10.1126/science. 1057103

Michalec, F. G., Holzner, M., Menu, D., Hwang, J.S., and Souissi, S. (2013). Behavioral responses of the estuarine calanoid copepod Eurytemora affinis to sub-lethal concentrations of waterborne pollutants. Aquat. Toxicol. 138-139, 129-138. doi: 10.1016/j.aquatox.2013.05.007

Murphy, E. and King, E. A. (2014). An assessment of residential exposure to environmental noise at a shipping port. Environ. Int. 63, 207-215. doi: 10.1016/j.envint.2013.11.001

Piña, B., and Barata, C. (2014). Encyclopedia of Toxicology, 3rd Edn. ed P. Wexler. New York, NY: Academic Press.

Robson, B. J. (in press). When do aquatic system models provide useful predictions, what is changing, and what is next? Environ. Model. Softw. doi: 10.1016/j.envsoft.2014.01.009

Setälä, O., Fleming-Lehtinen, V., and Lehtiniemi, M. (2014). Ingestion and transfer of microplastics 
in the planktonic food web. Environ. Pollut. 185, 77-83. doi: 10.1016/j.envpol.2013.10.013

Spruijt, P., Knol, A. B., Vasileiadou, E., Devilee, J., Lebret, E., and Petersen, A. C. (2014). Roles of scientists as policy advisers on complex issues: a literature review. Environ. Sci. Policy 40, 16-25. doi: 10.1016/j.envsci.2014.03.002

Sweet, B. (2013). Global CO2 Concentration Reaches 400 Parts Per Million - IEEE Spectrum. Available online at: http://spectrum.ieee.org/energywise/ energy/environment/global-co2-reaches-400-ppm (Accessed May 16, 2013).

WHO. (2012). State of the Science of Endocrine Disrupting Chemicals - 2012, eds Å. Bergman, J. J. Heindel, S. Jobling, K. A. Kidd, and R. T. Zoeller, 289.
Wiegleb, G., Bröring, U., Choi, G., Dahms, H.U., Kanongdate, K., Byeon, C.-W., et al. (2013). Ecological restoration as precaution and not as restitutional compensation. Biodivers. Conserv. 22, 1931-1948. doi: 10.1007/s10531-013-0518-0

Zenker, A., Cicero, M. R., Prestinaci, F., Bottoni, P., and Carere, M. (2014) Bioaccumulation and biomagnification potential of pharmaceuticals with a focus to the aquatic environment. J. Environ. Manage. 133, 378-387. doi: 10.1016/j.jenvman.2013.12.017

Conflict of Interest Statement: The author declares that the research was conducted in the absence of any commercial or financial relationships that could be construed as a potential conflict of interest.
Received: 28 March 2014; accepted: 03 May 2014; published online: 21 May 2014.

Citation: Dahms HU (2014) The grand challenges in marine pollution research. Front. Mar. Sci. 1:9. doi: 10.3389/fmars.2014.00009

This article was submitted to Marine Pollution, a section of the journal Frontiers in Marine Science.

Copyright $\odot 2014$ Dahms. This is an open-access article distributed under the terms of the Creative Commons Attribution License (CC BY). The use, distribution or reproduction in other forums is permitted, provided the original author(s) or licensor are credited and that the original publication in this journal is cited, in accordance with accepted academic practice. No use, distribution or reproduction is permitted which does not comply with these terms. 